\title{
Studies of Local Horizontal-Vertical Betatron Coupling in the Fermilab Main Ring
}

\author{
G. Bourianoff, N. Sun, and G. P. Tsironis \\ Superconducting Super Collider Laboratory* \\ 2550 Beckleymeade Ave. \\ Dallas, TX 75237 \\ and \\ I. Kourbanis \\ Fermi National Accelerator Laboratory \\ P. O. Box 500, MS 341 \\ Batavia, IL 60510
}

September 1993

*Operated by the Universities Research Association, Inc., for the U.S. Department of Energy under Contract No. DE-AC35-89ER40486.

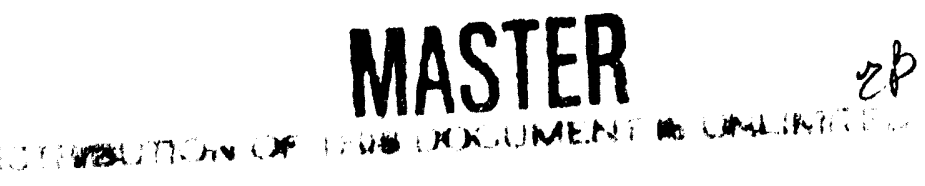




\title{
Studies of Local Horizontal-Vertical Betatron Coupling in the Fermilab Main Ring
}

\author{
G. Bourianoff, N. Sun, G. P. Tsironis
}

and

I. Kourbanis

\begin{abstract}
We give preliminary evidence that local horizontal-vertical betatron coupling can be detected using digital signal processing techniques. We use the Fermilab Main Ring to perform a number of local detection experiments using UDAS. For the data analysis we use straightforward FFT's as well as more sophisticated parametric estimation techniques. We show that the latter enables the detection of local coupling with some accuracy.
\end{abstract}




\subsection{INTRODUCTION}

Transverse betatron coupling has a number of undesirable effects in the regular operation of an accelerator. It can lead to, among other things, emittance growth and decrease of the dynamic aperture of the machine. Traditionally, the effects of coupling are compensated on the average by adjusting special skew quads included in the lattice for that purpose. The method used is that of "tune coincidence," which consists of the following steps: (a) We bring the tunes to close proximity by changing the currents in the regular quads, (b) minimize the distance between the tunes (in tune space) by varying the currents of the skew quads, and (c) once an absolute minimum in the tune distance is established with the skew quads, change the currents in the regular quads to bring the tunes back in their original position. This procedure, which has a typical duration of about one hour in proton machines, is not very practical for the Superconducting Super Collider (SSC). Furthermore, the coupling correction accomplished is an average or global one, viz. there can still be large local variations in coupling even if the coupling has been corrected globally. For the SSC local coupling correction has been proposed; the latter will enable a uniform reduction of the coupling around the ring and increase the reliability of the operation. A necessary condition for local coupling correction is an accurate local coupling determination. The latter can only happen if a number of different conditions on tune measurements can be fulfilled. Tune measurements have to be accurate and reliable; this necessitates a reliable and fast data acquisition system. They have to be nondestructive to the regular machine operation and thus they must be nonintrusive. Accurate measurements of the power into each oscillation mode must be obtained and from them local coupling determinations should be obtained.

The problem of the local ccupling correction is quite a complicated one. It involves a harmonious interaction among theory, simulations and real experiments. From theory we obtain the desired expressions that give the coupling coefficients locally. We use the experiment to test the ideas and simulations to check both. In the present paper, we will primarily focus on the experimental aspects of the preliminary coupling studies performed so far and the numerical techniques used to obtain the desired quantities. In a follow up paper, we will present theoretical studies that assist the physical understanding of the situation and will be used in the ultimate implementation of the decoupling procedures.

\subsection{LOCAL MEASUREMENTS WITH A NOISE SOURCE}

Coupling experiments have ber $n$ performed in two accelerators, in HERA, and also in the Fermilab Main Ring. The results from the former have been reported elsewhere. ${ }^{1}$ In this paper, we focus on the preliminary studies done in the Fermilab Main Ring. The motivations for these studies were the following: (a) demonstrate that local tune measurements are possible by applying a noise source on the beam to excite the betatron frequencies, (b) use local tune information and change the skew quad currents to observe detectable changes in the local coupling, (c) perform data analysis on the data obtained from the experiments, and (d) experiment with different signal processing techniques to find the optimal way of extracting thn.e coupling information contained in the data obtained in (a) and (b). Once this first phase is completed, use the experience and knowledge obtained to perform a local coupling detection and correction experiments. In the latter, experimental results from the local coupling measurements are fed into the theoretical formulas to predict the skew quad strengths necessary for local coupling correction.

For the experiments at Fermilab we used the Unix Data Acquisition System (UDAS). ${ }^{2}$ We had at our disposal four fully functional Beam Position Monitors (BPMs) located in the F section of the Main Ring, H46, H49 (horizontal), V45 and V49 (vertical). Turn-by-turn data were channeled from the BPMs to a Lecroy 
digitizer and subsequently stored in shared memory in a Sun workstation. The turn-by-turn data can be viewed in real time and also stored for analysis.

To obtain tune measurements from turn-by-turn data in times other than during injection, detectable betatron oscillations must be induced in the beam. This can happen with a sudden kick (or ping) or by a continuous beam disturbance with a noise source. In the first phase of our studies we applied a chirped noise source in the Main Ring dampers and perturbed the beam in a continuous fashion with a strength that led to no beam degradation. The essence of this experiment is to demonstrate that tune information can be obtained in a totally nonintrusive, parasitic fashion. For the chirp experiment we used an HP synthesizer with a central frequency equal to $19.5 \mathrm{kHz}$ and a frequency span $3 \mathrm{kHz}$. We varied the voltage (amplitude) of the sine wave and the duration of the chirp. Using signal analysis techniques (most work was done with the software package MATLAB) on the raw data we obtained the desired tune information. Results from these measurements are reported in Reference 1 .

\subsection{LOCAL MEASUREMENTS WITH A BEAM PING}

A sudden electrostatic kick induces betatron oscillations in the beam that can be observed in an analysis of the turn-by-turn data. In an ideal machine with no coupling, a horizontal/vertical ping leads to horizontal/vertical motion, respectively. In a coupled machine, a ping in one plane induces oscillations in both planes. If the ping is sufficiently strong, both "horizontal-like" and "vertical-like" oscillations are visible in either the "horizontal" or "vertical" turn-by-turn data. It has been suggested" that accurate measurement of the tune information contained in the data can lead to a complete linear coupling determination. If data are observed in various ring locations, local coupling determination is possible.

Four distinct coupling coefficients enter in the local coupling theory; two are related to the inclination of the local eigenplanes and the rest are proportional to the ratio of the power of oscillations in the two plane. Presently, we will ignore the local eigenplane tilt coefficients and focus of the other two. The latter can be determined straightforwardly for the ping data in the following fashion: Let us assume that we have a pair of BPMs in a given ring location, one of which is detecting the local horizontal beam oscillations and the other the vertical one. A horizontal ping will induce horizontal as well as (because of coupling) vertical betatron oscillations which will be in principle detected by both BPMs. In the horizontal BPM most power will be in the horizontallike betatron motion whereas in the vertical one a small fraction of the input power will appear in the horizontallike oscillations. It has been shown ${ }^{3}$ that the coupling coefficient associated with this observation scheme is proportional to the ratio of the powers for the same type of oscillation (same tune) determined by the vertical and horizontal BPMs, respectively. In other words, if we observe signal in two frequencies, say $\omega_{-}$and $\omega_{+}$in both BPMs and the former frequency is the horizontal-like one whereas the latter is the vertical-like one, then the coupling coefficients are proportional to the ratio of the power in $\omega_{-}\left(\omega_{+}\right)$measured in the two BPMs.

For the experiments in the Fermilab Main Ring we used four BPMs two of which constituted a pair measuring horizontal and vertical signal in the same lattice location. The beam was pinged horizontally and vertically, turn-by-turn signals were extracted, Fourier analyzed and the relevant coupling coefficients determined. Subsequently, the amount of local coupling was altered by making changes in the skew quadrupole magnets of the Main Ring. The latter are divided in three families of six quads each that are individually powered but linked in the control software together. The software does not allow for an individual control of the currents of each family of skew quads separately. On the other hand, since the effect of skew quad in the beam is the introduction of driving terms in the betatron equations of motion, it is appropriate to use a "phasor" language to describe these effects. ${ }^{4}$ The skew quad effects are controlled by varying the "angle" and the "magnitude" of the total "skew quad phasor." By adjusting these two quantities appropriately, the local values of the coupling coefficients can change. 
In this phase of the experiment we attempted to change the angle-amplitude coordinates of the skew quads in such a way as to chserve detectable changes in the local coupling coefficients. We took three sets of data:

TABLE 1. DATA SETS FOR COUPLING EXPERIMENTS.

\begin{tabular}{|c|c|c|}
\hline Data Sets & Ping & Changes \\
\hline$b$ & Horizontal & Skew Angle \\
\hline$c$ & Horizontal & Skew Amplitude \\
\hline$d$ & Vertical & Skew Angle \\
\hline
\end{tabular}

All data were placed in shared memory, subsequently put in SDS-type files and analyzed later.

\subsection{LOCAL COUPLING AND DIGITAL SIGNAL ANALYSIS}

For the analysis of the data we used two different approaches: (a) Fast Fourier Transforms and (b) Parametric Estimation Techniques. For the first approach we simply did an FFT of the appropriate segment of the turn-byturn data and subsequently evaluated the coupling coefficients. We then obtained the desired quantities as a function of the skew angle and skew amp litude. In the results we observed substantial variations of the coupling coefficients for different angles. These variations did not enable an unequivocal determination of a minimum in the coupling coefficients for some skew angle which was consistent in all data sets available. The basic reason for this "unexpected" outcome is the presence of noise in the signals.

We then used linear regression techniques for the determination of the coupling coefficients. We did the anal$y$ sis of the data sets with the software package MATLAB. For this analysis we made the following basic assumption: When the beam is disturbed (pinged) in one plane (say horizontal) then the turn-by-turn data from that plane are considered independent (or input) data, whereas the data from the other plane are thought of as dependent (or output) data. This assumption enables us to study the power transfer from one plane to the other with a simple ARX model ${ }^{5}$ and obtain the desired information from the study of its transfer function. We should point out here, that the adoption of an ARX-model is not entirely justified since the latter assumes one specific "direction" in the power transfer whereas, in our case, there is certainly some feedback from the assumed dependent variables to the assumed independent ones. A more careful analysis would require a complete VARMA model which incorporates feedback terms in its assumptions.

The "System Identification Toolbox" of MATLAB has optimization capabilities in terms of finding the best ARX model to analyze our tum-by-turn data. After some testing we concluded that the best model for our data is a an ARX $[15,15,1]$ one, viz. one which takes into account 15 lags both for the dependent and independent variables. The real part of the transfer function for this model showed distinct peaks at some frequencies. The latter were exactly the same tune frequencies obtained from the FFTs. By forming the coupling radii we obtained an estimate for the local coupling as a function of the skew quad variables. Turn-by-turn data, FFTs as well as the results of the analysis are presented in Figures 1-3.

\subsection{CONCLUSIONS}

In this preliminary experimental work we focussed on two issues. The first was related with the tune information as a result of weak beam perturbations with a noise source. We found that it is indeed possible to obtain tune data and coupling coefficients using this approach. The second goal was to obtain detectable coupling variations for different skew quad settings. We found that this is also possible but the extraction of the coupling 
information can be elusive unless proper analysis tools are used. We discovered that, in some cases, regression analysis techniques are necessary for obtaining results with reasonable accuracy. The next step in this work will be in the direction of: (a) more complete exploration of coupling measurements for a broader regime of skew quad currents, and (b) effort for correction of local coupling based on the information obtained from the coupling measurements.

\section{REFERENCES}

1. G. Bourianoff, "Operational Decoupling of the SSC," SSCL preprint.

2. I. Kourbanis, et al., "The Unix Data Acquisition System," 1991 IEEE Part. Accel. Conf. Proceedings,

p. 1302, San Francisco (1991).

3. R. Talman, "Single Particle Motion," Notes for Joint US-CERN School on Beams, Capri (1998).

4. R. Gerig,"Detection and Compensation of Transverse Resonances," AIP Proceedings, vol.184, p. 1929 (1989).

5. L. Ljung, System Identification, Prentice-Hall, Englewood Cliffs (1987). 
a.
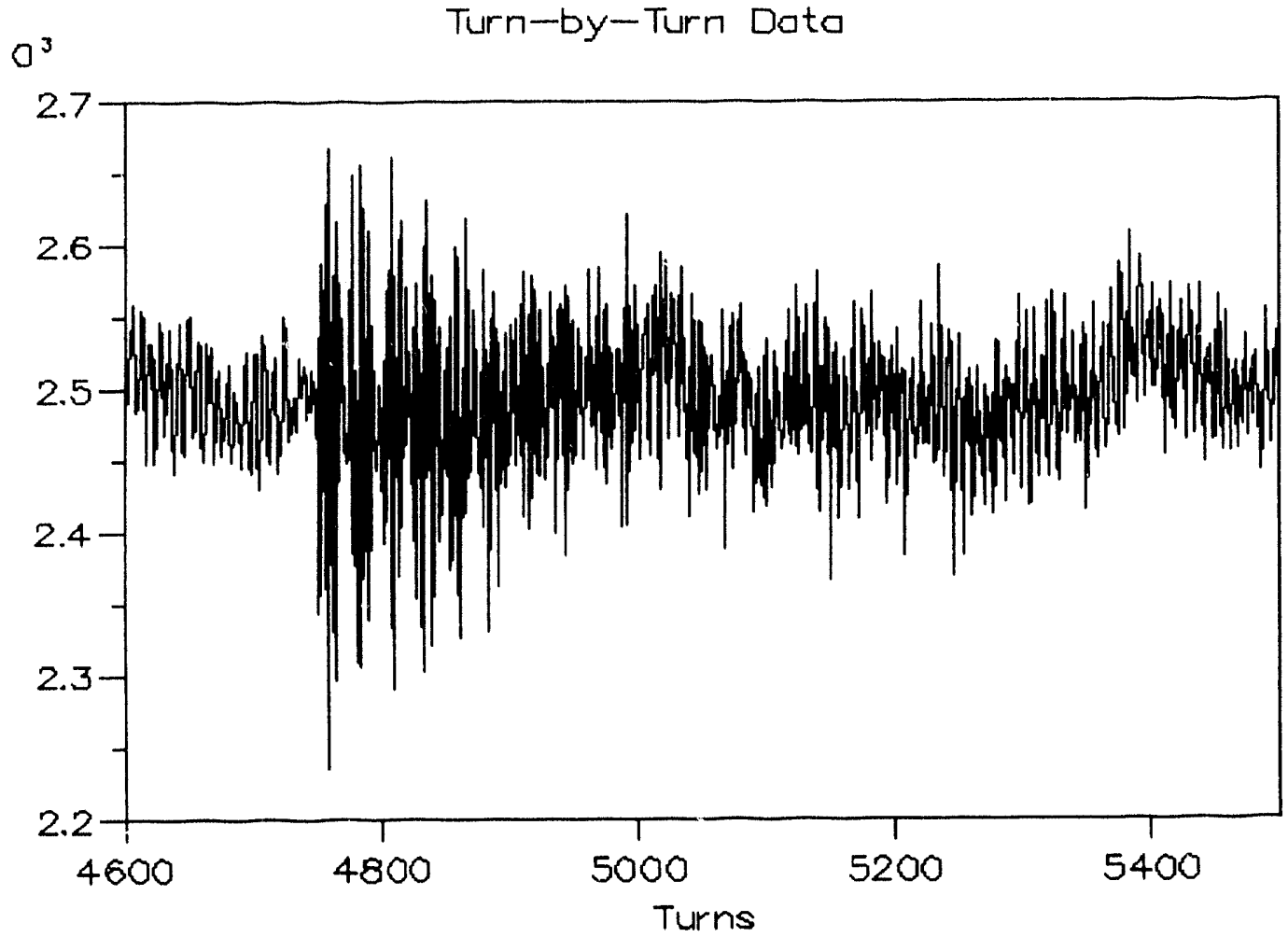

b.

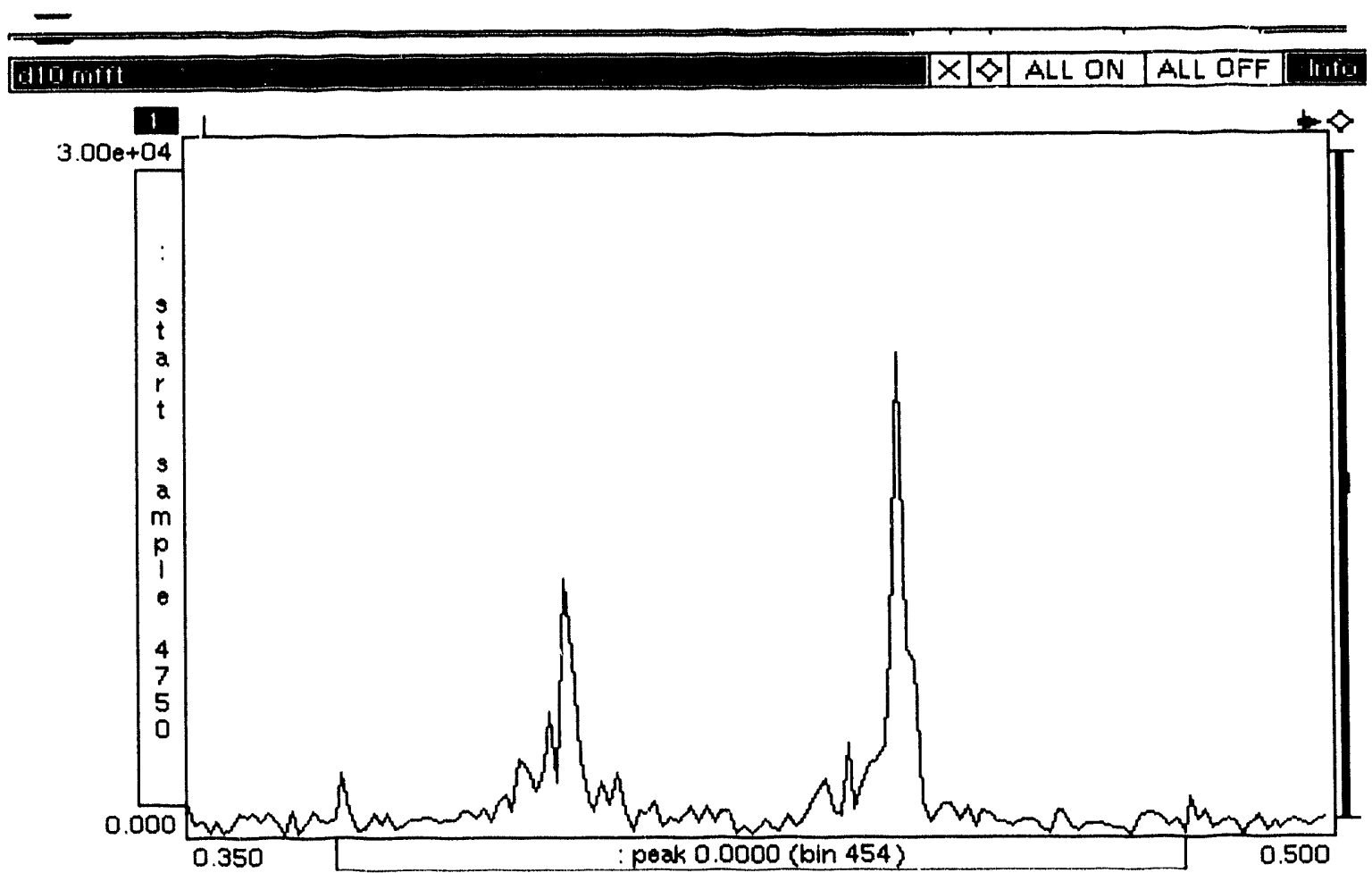

Figure 1. (a) Raw Turn-by-turn Data Obtained in a Horizontal BPM After a Ping in the Horizontal Plane. (b) FFT of the raw data showing the horizontal (smaller frequency) and vertical tunes of the Main Ring. 
c

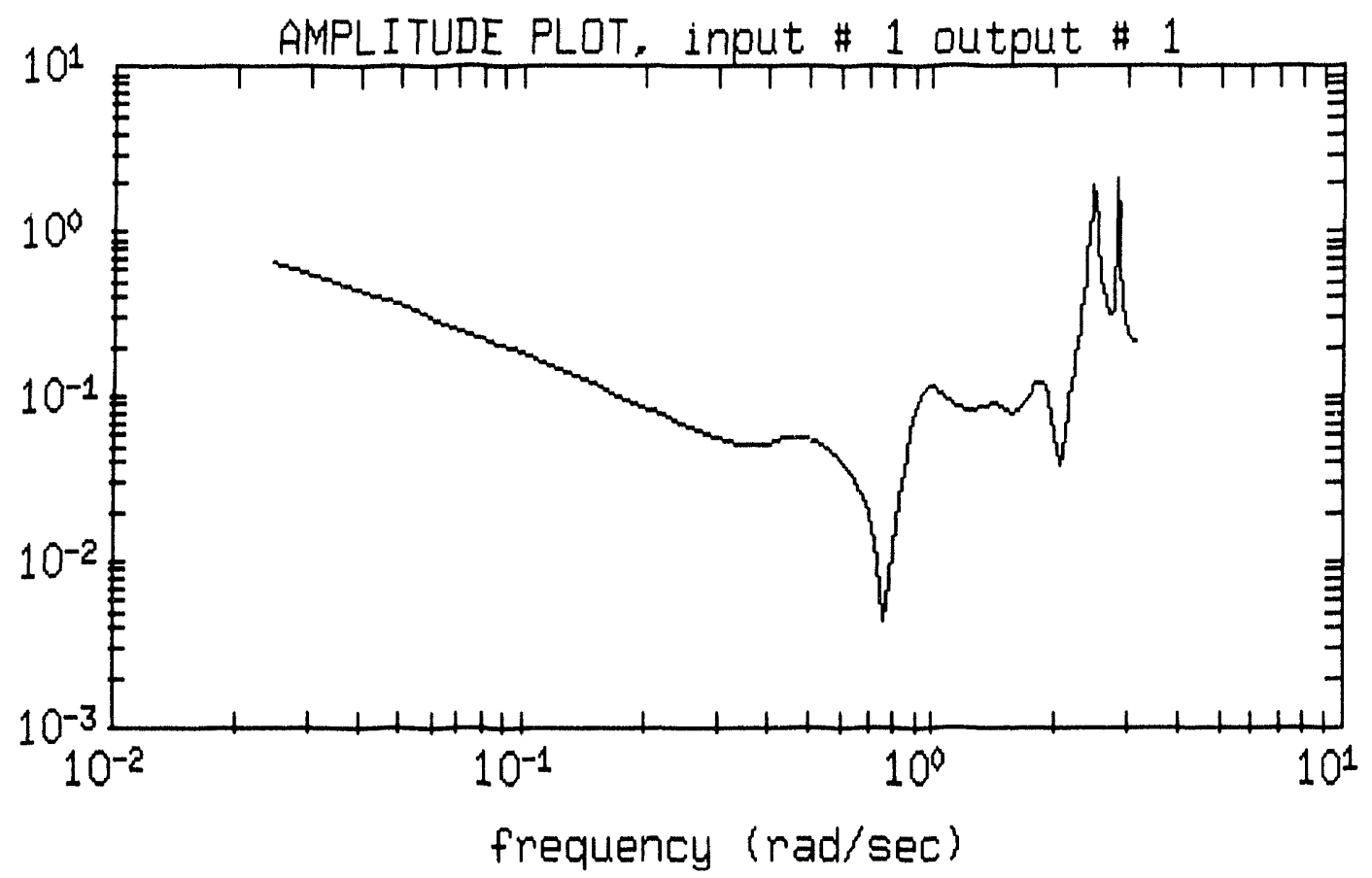

Figure 1. (c) Ratio of the Amplitudes of the Vertical-horizontal Signals as a Function of Frequency Using the ARX Model. The peaks on the right end of the plot are proportional to the local coupling of the machine measured at the BPM location.

a.

B Datset

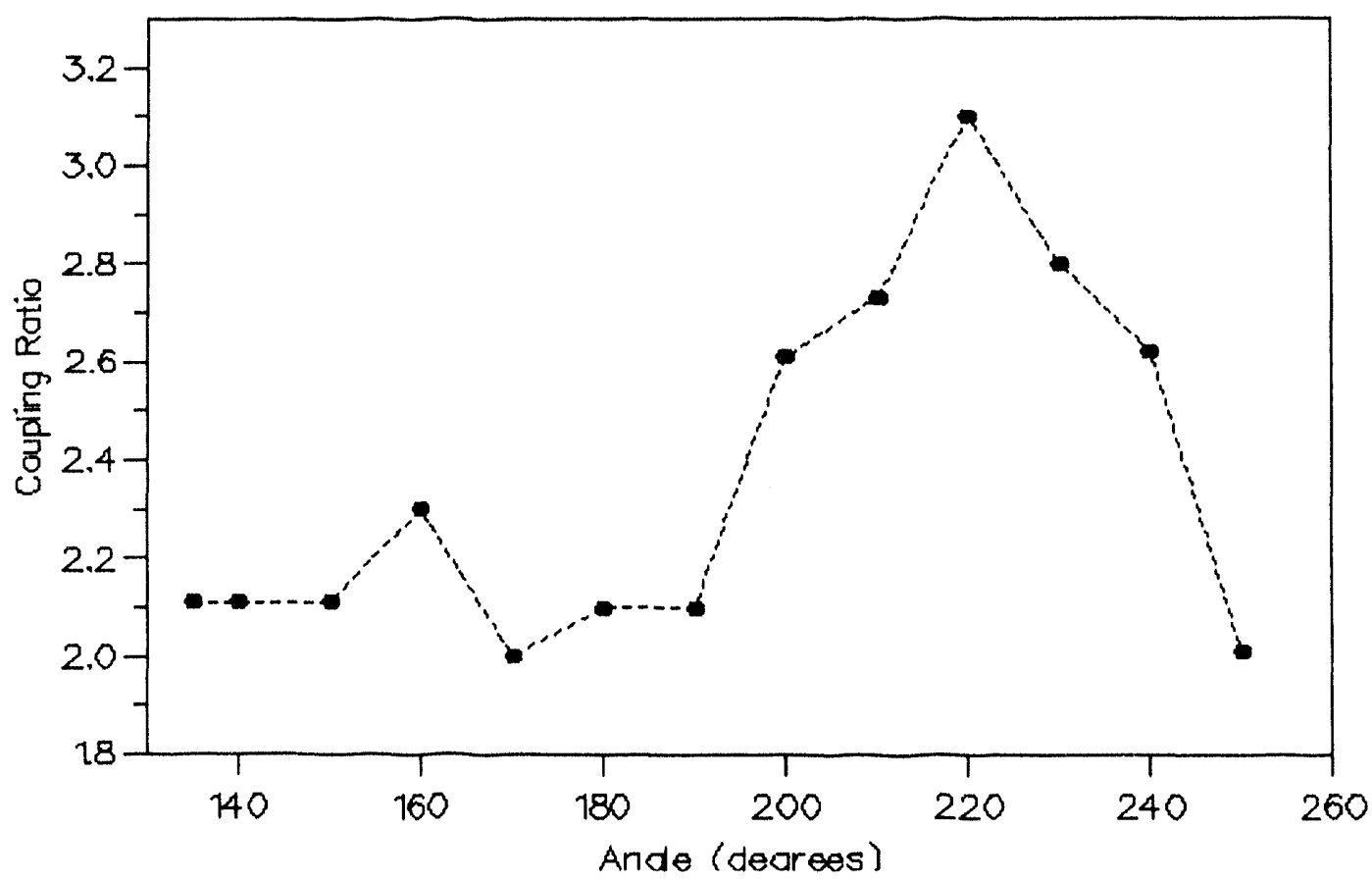

Figure 2. Coupling Ratios, 1.e., Off-plane over On-Plane Ratios at a Given Tune, as a Function of the Phasor Angle After (a) a Horizontal Ping. (On-plane refers to plane of the ping.) 
b.

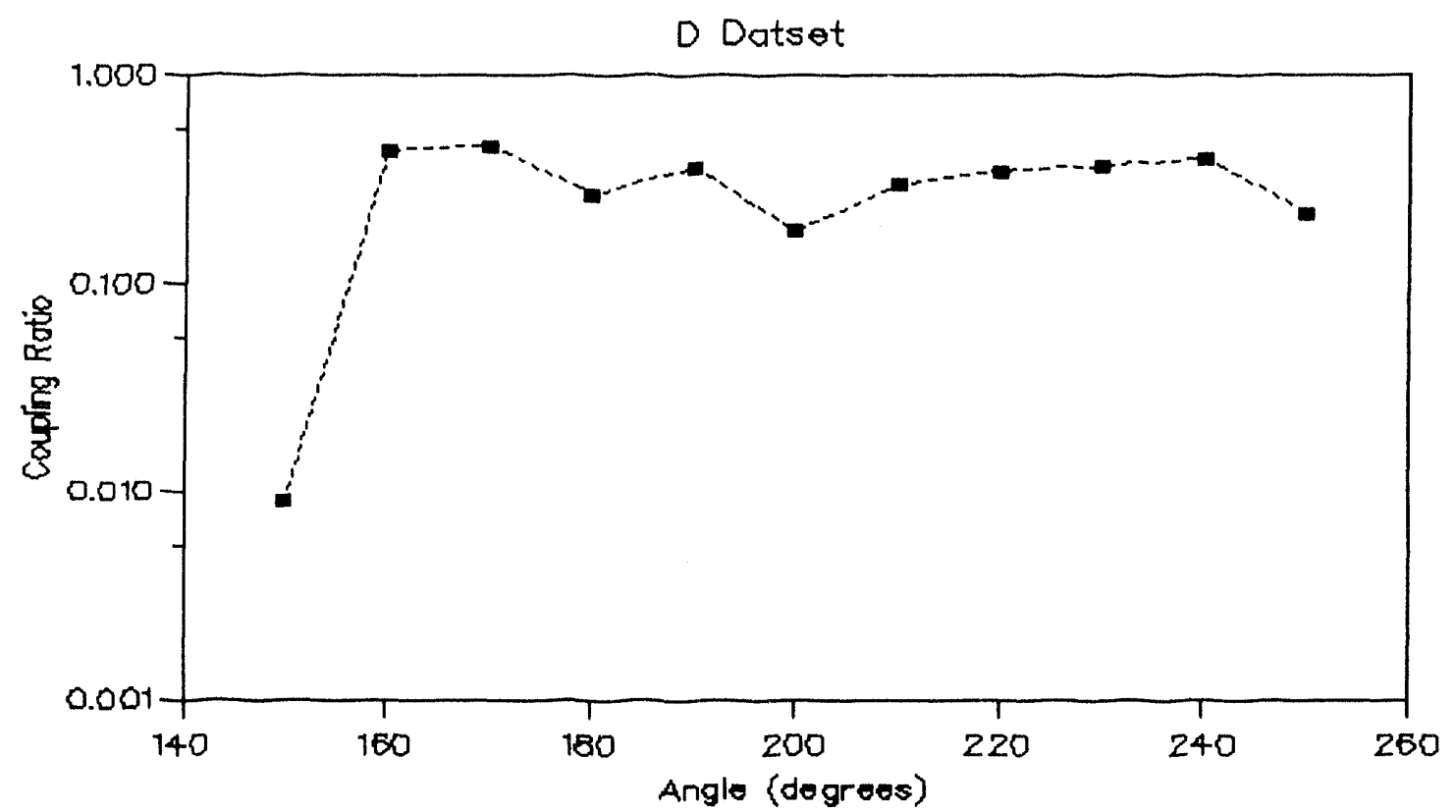

Figure 2. (b) A Vertical Ping (in Arbitrary Units).

c.

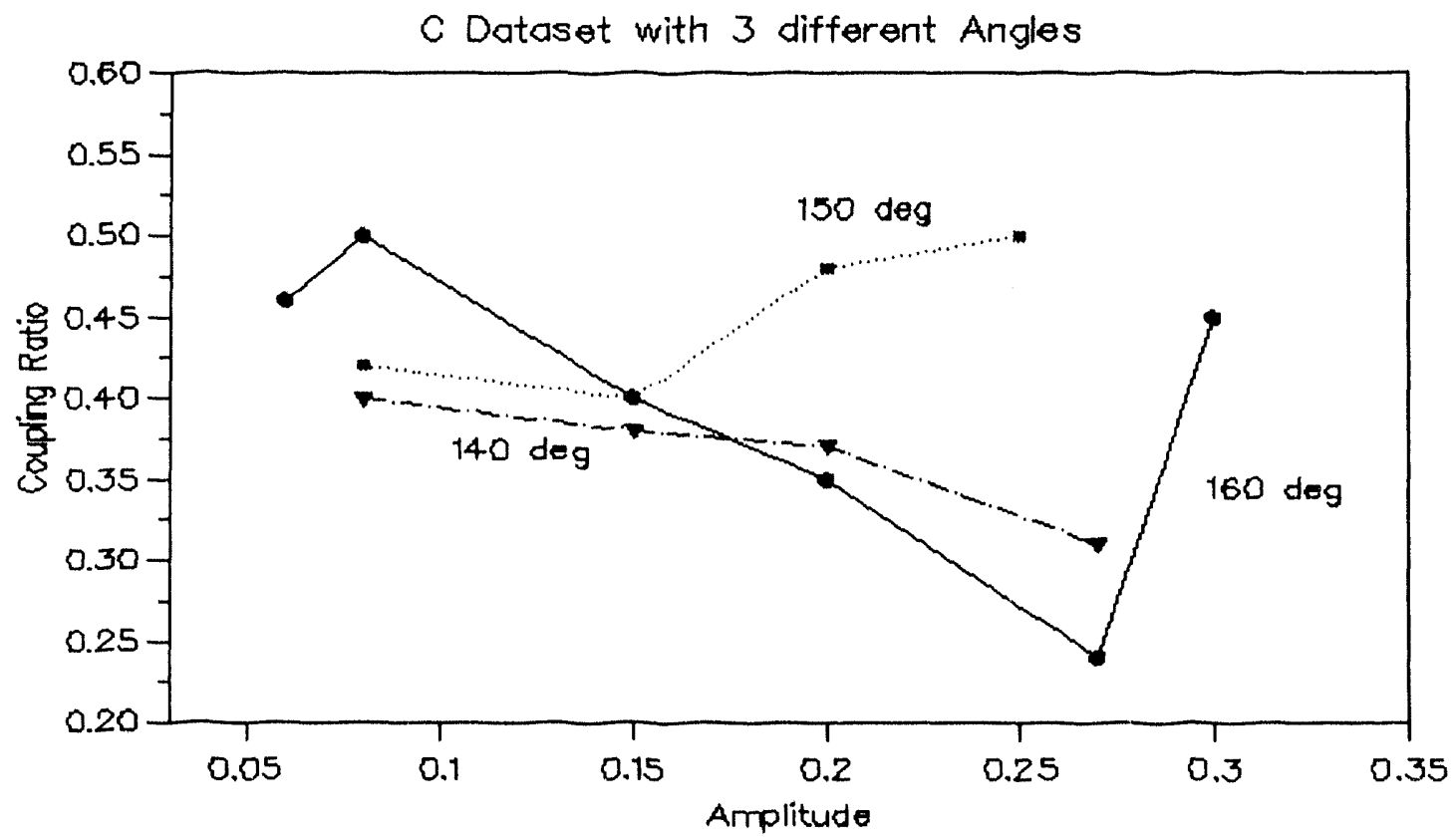

Figure 3. Coupling Ratios as a Function of Phasor Amplitude for Different Phasor Angles. The angles are near the minimum coupling angle of the prevlous figure. 

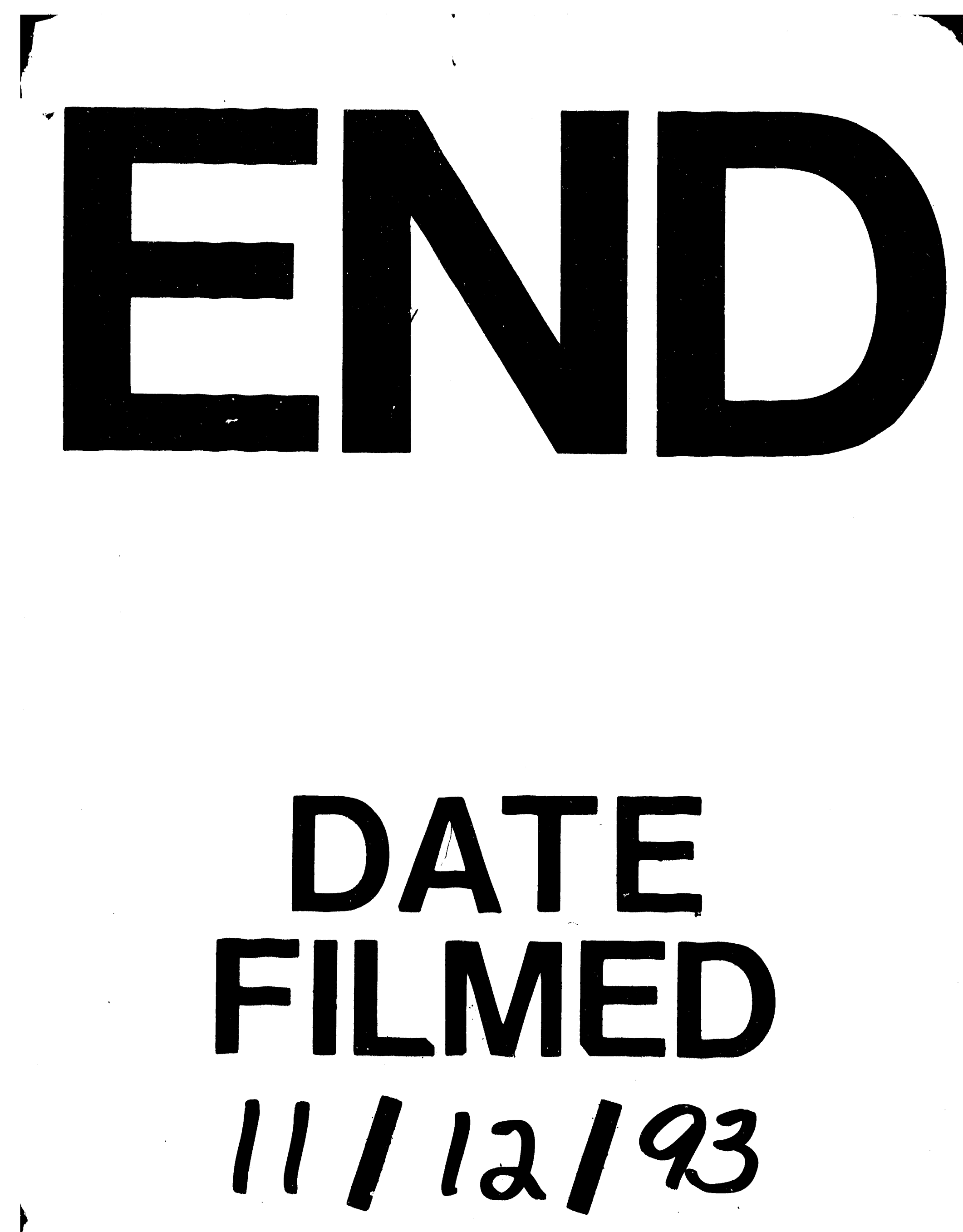

1 
$-\ldots$

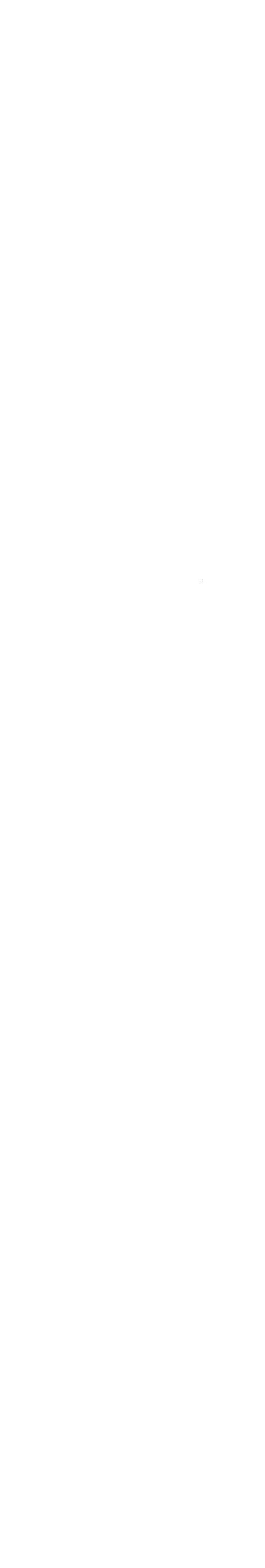

